\title{
Preparation of cellulose nanofibers with hydrophobic surface characteristics.
}

\begin{abstract}
The aim of this study was to develop cellulose nanofibers with hydrophobic surface characteristics using chemical modification. Kenaf fibers were modified using acetic anhydride and cellulose nanofibers were isolated from the acetylated kenaf using mechanical isolation methods. Fourier transform infrared spectroscopy (FTIR) indicated acetylation of the hydroxyl groups of cellulose. The study of the dispersion demonstrated that acetylated cellulose nanofibers formed stable, well-dispersed suspensions in both acetone and ethanol. The contact angle measurements showed that the surface characteristics of nanofibers were changed from hydrophilic to more hydrophobic when acetylated. The microscopy study showed that the acetylation caused a swelling of the kenaf fiber cell wall and that the diameters of isolated nanofibers were between 5 and $50 \mathrm{~nm}$. X-ray analysis showed that the acetylation process reduced the crystallinity of the fibers, whereas mechanical isolation increased it. The method used provides a novel processing route for producing cellulose nanofibers with hydrophobic surfaces.
\end{abstract}

Keyword: Kenaf; Nanofibers; Acetylation; Chemical characterization; Structural characterization. 